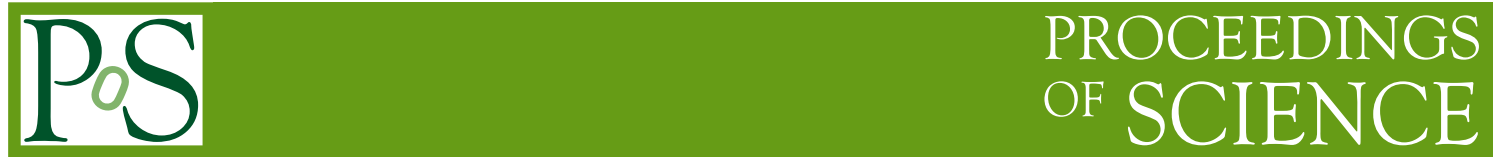

\title{
Nuclear forces on the lattice
}

\section{Silas Beane*}

University of New Hampshire

E-mail: silas@physics.unh.edu

Recent studies by the NPLQCD collaboration of hadronic interactions using lattice QCD are reviewed, with an emphasis on a recent calculation of meson-baryon scattering lengths. Ongoing high-statistics calculations of baryon interactions are also reviewed. In particular, new insights into the signal/noise problems that plague correlation functions involving baryons are discussed.

6th International Workshop on Chiral Dynamics

July 6-10 2009

Bern, Switzerland

\footnotetext{
*Speaker.
} 


\section{Introduction}

Quantum Chromodynamics (QCD) — the underlying theory of the strong interactions- when combined with the electroweak interactions, is responsible for all of nuclear physics, from the structure of light nuclei to the most complex nuclear reactions. Presently lattice QCD is the only known method which allows one to compute properties of hadrons and nuclei from first principles. While lattice QCD provides highly-accurate predictions and postdictions of hadronic properties like masses and decay constants, the study of hadronic interactions, like two-body scattering and three-body interactions is still in a development phase. The NPLQCD collaboration has calculated the interactions between many of the lowest-lying baryons and mesons, including $\pi^{+} \pi^{+}, K^{+} \pi^{+}$, $K^{+} K^{+}$, meson-baryon, nucleon-nucleon, and hyperon-nucleon scattering as well as multi-meson systems (much of this work is reviewed in Ref. [1]). It is noteworthy that the $\pi^{+} \pi^{+}$scattering length has been calculated to percent-level accuracy at the physical point [2]. Part of the reason behind this accuracy is that QCD correlation functions involving Goldstone bosons are subject to powerful chiral symmetry constraints. Since current lattice calculations are carried out at unphysical quark masses, these constraints play an essential role in extrapolating the lattice data to the physical quark masses, as well as to the infinite volume, and continuum limits. Chiral perturbation theory $(\chi-\mathrm{PT})$ is the optimal method for implementing QCD constraints due to chiral symmetry, and in essence, provides an expansion of low-energy S-matrix elements in quark masses and powers of momentum [3].

In contrast to the purely mesonic sector, recent studies of baryon-baryon interactions - the paradigmatic nuclear physics process - have demonstrated the fundamental difficulty faced in making predictions for baryons and their interactions $[4,5]$. Unlike the case with mesons, correlation functions involving baryons suffer an exponential degradation of signal/noise at large times [6]. Furthermore, while baryon interactions are constrained by QCD symmetries like chiral symmetry, the constraints are not nearly as powerful as when there is at least one pion or kaon in the initial or final state. For instance, there is no expectation that the baryon-baryon scattering lengths vanish in the chiral limit as they do in the purely mesonic sector. To make matters worse, in s-wave nucleonnucleon scattering, the interactions are enhanced due to the close proximity of a non-trivial fixed point of the renormalization group, which drives the scattering lengths to infinity, thus rendering the effective field theory description of the interaction non-perturbative [7].

In this proceedings I will review the most recent progress made in the description of hadronic scattering and interactions. Given the contrast in difficulty between the purely mesonic and purely baryonic sectors described above, it is clearly of great interest to perform a lattice QCD investigation of the simplest scattering process involving at least one baryon: meson-baryon scattering. The first fully-dynamical study of meson-baryon scattering has been carried out recently in Ref. [8] and will be reviewed. I will also review other recent work $[9,10]$ by the NPLQCD collaboration, which has performed very-high statistics calculations of few-body baryon systems that offer new insights into the signal/noise problem, and allow a first glimpse of the baryon number $B=3$ sector.

\section{Signal to Noise Estimates}

As is well known [6], very general field-theoretic arguments allow a robust estimate of the noise to 
signal ratio of hadronic correlation functions calculated on the lattice. With an eye towards lattice QCD attempts to describe nuclei, it is worth briefly noting the fundamental difference between lattice-measured correlation functions involving mesons and baryons. As an example, consider the noise to signal ratio of a correlation function involving $n$ pion fields, where the small interaction is neglected,

$$
\frac{\sigma(t)}{\langle\theta(t)\rangle} \sim \frac{\sqrt{\left(A_{2}-A_{0}^{2}\right)} e^{-n m_{\pi} t}}{\sqrt{N} A_{0} e^{-n m_{\pi} t}} \sim \frac{1}{\sqrt{N}} .
$$

Here $\langle\theta(t)\rangle$ is the correlation function, $\sigma(t)$ is the variance and the $A_{i}$ are amplitudes. It is noteworthy that in this ratio, the time dependence of the variance mirrors the time dependence of the correlator itself. One therefore concludes that correlators involving arbitrary numbers of pions have time-independent errors, as is indeed observed in lattice calculations. This of course renders the study of mesonic correlators quite pleasurable (from the statistical perspective).

The baryons provide a more disturbing story; consider the noise to signal ratio for a proton correlation function:

$$
\frac{\sigma(t)}{\langle\theta(t)\rangle} \sim \frac{\sqrt{A_{2}} e^{-\frac{3}{2} m_{\pi} t}}{\sqrt{N} A_{0} e^{-m_{p} t}} \sim \frac{1}{\sqrt{N}} e^{\left(m_{p}-\frac{3}{2} m_{\pi}\right) t}
$$

Here the variance is dominated by the three-pion state rather than by the proton, and therefore the noise to signal ratio of the proton correlator grows exponentially with time. More generally, for a system of $A$ nucleons, the noise to signal ratio behaves as

$$
\frac{\sigma(t)}{\langle\theta(t)\rangle} \sim \frac{1}{\sqrt{N}} e^{A\left(m_{p}-\frac{3}{2} m_{\pi}\right) t}
$$

Therefore the situation worsens as one adds nucleons. Indeed one can show that the theoretical expectation from eq. 2.3 compares favorably with $A=2$ data calculated by the NPLQCD collaboration [11].

The estimates given above, which follow from very general field theoretic arguments, naively indicate that nucleon and nuclear physics require exponentially more resources than meson physics to achieve the same level of accuracy. While these general results are somewhat discouraging to efforts to extract baryon interactions from the lattice, one should keep in mind that the arguments are rigorous only in the asymptotic, infinite time, limit. And, indeed, as we shall see below, there can be a region of intermediate times where these arguments do not apply, rendering the problem much less severe than these arguments suggest.

\section{Meson-meson interactions}

Here I will briefly review a calculation of the $I=2$ pion-pion $(\pi \pi)$ scattering length, as it serves as a benchmark calculation with an accuracy that can only be aspired to at present for other systems. Due to the chiral symmetry of QCD, pion-pion $\pi \pi$ scattering at low energies is the simplest and best-understood hadron-hadron scattering process. The scattering lengths for $\pi \pi$ scattering in the s-wave are uniquely predicted at leading order in chiral perturbation theory $(\chi$-PT) [12]:

$$
m_{\pi} a_{\pi \pi}^{I=0}=0.1588 ; m_{\pi} a_{\pi \pi}^{I=2}=-0.04537,
$$


at the charged pion mass. While experiments do not provide stringent constraints on the scattering lengths, a determination of s-wave $\pi \pi$ scattering lengths using the Roy equations has reached a remarkable level of precision $[13,14]$ :

$$
m_{\pi} a_{\pi \pi}^{I=0}=0.220 \pm 0.005 ; m_{\pi} a_{\pi \pi}^{I=2}=-0.0444 \pm 0.0010
$$

The Roy equations [15] use dispersion theory to relate scattering data at high energies to the scattering amplitude near threshold. At present, lattice QCD can compute $\pi \pi$ scattering only in the $I=2$ channel as the $I=0$ channel contains disconnected diagrams. It is of course of great interest to compare the precise Roy equation predictions with lattice QCD calculations. Figure 1 summarizes theoretical and experimental constraints on the s-wave $\pi \pi$ scattering lengths [14]. It is clearly a strong-interaction process where theory has outpaced the very-challenging experimental measurements.

The only existing fully-dynamical lattice QCD prediction of the $I=2 \pi \pi$ scattering length involves a mixed-action lattice QCD scheme of domain-wall valence quarks on a rooted staggered sea. Details of the lattice calculation can be found in Ref. [2]. The scattering length was computed at pion masses, $m_{\pi} \sim 290 \mathrm{MeV}, 350 \mathrm{MeV}, 490 \mathrm{MeV}$ and $590 \mathrm{MeV}$, and at a single lattice spacing, $b \sim 0.125 \mathrm{fm}$ and lattice size $L \sim 2.5 \mathrm{fm}$ [2]. The physical value of the scattering length was obtained using two-flavor mixed-action $\chi$-PT which includes the effect of finite lattice-spacing

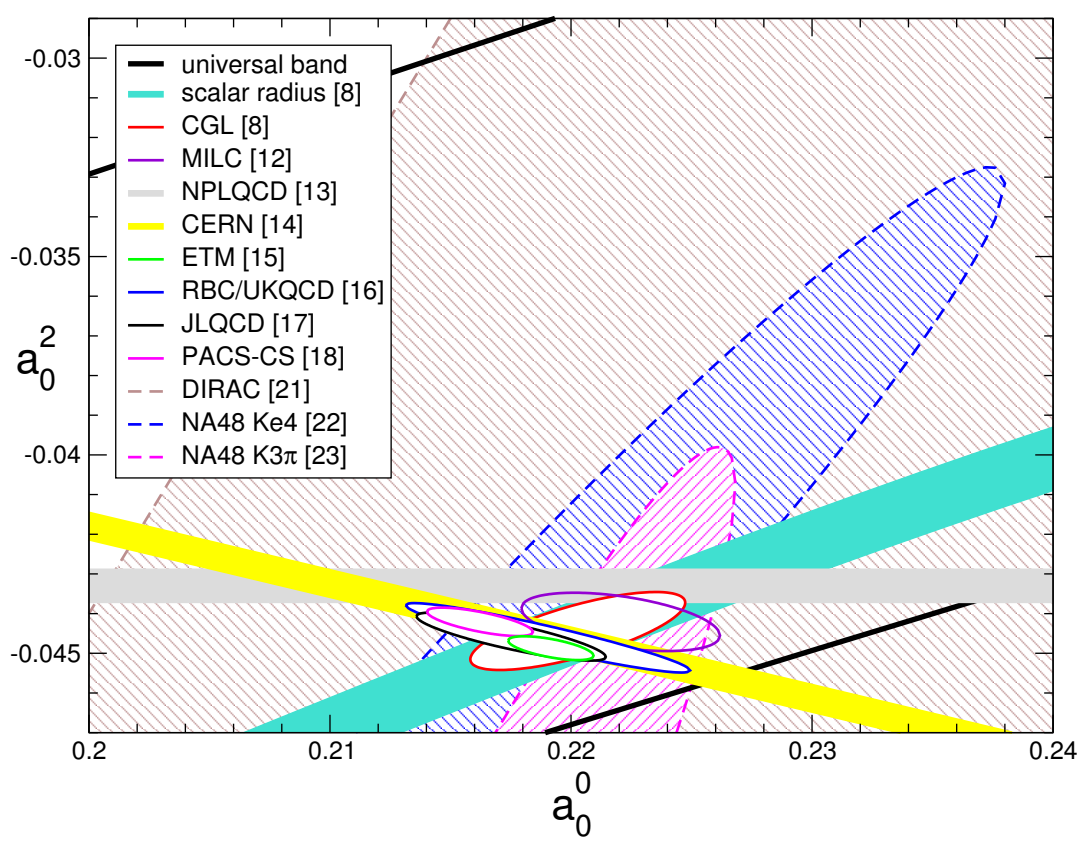

Figure 1: The state of threshold s-wave $\pi \pi$ scattering. Noteworthy are the red ellipse from the Roy equation analysis and the grey band from the direct lattice QCD calculation of the $I=2$ scattering length, as discussed in the text. 
artifacts to $\mathscr{O}\left(m_{\pi}^{2} b^{2}\right)$ and $\mathscr{O}\left(b^{4}\right)[16]$. The final result is:

$$
m_{\pi} a_{\pi \pi}^{I=2}=-0.04330 \pm 0.00042
$$

where the statistical and systematic uncertainties have been combined in quadrature. Notice that $1 \%$ precision is claimed in this result. The agreement between this result and the Roy equation determination is a striking confirmation of the lattice methodology, and a powerful demonstration of the constraining power of chiral symmetry in the meson sector. It would clearly be of great interest to see other (fully-dynamical) lattice QCD calculations of the s-wave $\pi \pi$ scattering lengths using different types of fermions. The $K^{+} K^{+}$and $\pi^{+} K^{+}$scattering lengths have also been computed by the NPLQCD collaboration. I refer the interested reader to Ref. [1] for details.

\section{Meson-Baryon Interactions}

Pion-nucleon scattering has long been considered a paradigmatic process for the comparison of $\chi$ PT and experiment. To this day, controversy surrounds determinations of the pion-nucleon coupling constant and the pion-nucleon sigma term. The $K^{-} n$ interaction is important for the description of kaon condensation in the interior of neutron stars [17], and meson-baryon interactions are essential input in determining the final-state interactions of various decays that are interesting for standardmodel phenomenology (See Ref. [18] for an example). In determining baryon excited states on the lattice, it is clear that the energy levels that represent meson-baryon scattering on the finite-volume lattice must be resolved before progress can be made regarding the extraction of single-particle excitations.

While pion-nucleon scattering is the best-studied meson-baryon process, both theoretically and experimentally, its determination on the lattice is computationally prohibitive since it involves annihilation diagrams. At present only a few limiting cases that involve these diagrams are being investigated [19]. Combining the lowest-lying $S U(3)$ meson and baryon octets, one can form five meson-baryon elastic scattering processes that do not involve annihilation diagrams: $\pi^{+} \Sigma^{+}, \pi^{+} \Xi^{0}$, $K^{+} p, K^{+} n$, and $\bar{K}^{0} \Xi^{0}$. (Note that $\bar{K}^{0} \Sigma^{+}$has the same quantum numbers as $\pi^{+} \Xi^{0}$.) Three of these processes involve kaons and therefore are, in principle, amenable to an $S U(3)$ heavy-baryon $\chi$-PT (HB $\chi-\mathrm{PT})$ analysis [20] for extrapolation. The remaining two processes involve pions interacting with hyperons and therefore can be analyzed in conjunction with the kaon processes in $S U(3)$ $\mathrm{HB} \chi$-PT, or independently using $S U(2) \mathrm{HB} \chi$-PT. Below we will review recent work in Ref. [8] which has carried out this analysis.

The calculations of Ref. [8] were performed predominantly with the coarse MILC lattices with a lattice spacing of $b \sim 0.125 \mathrm{fm}$, and a spatial extent of $L \sim 2.5 \mathrm{fm}$. On these configurations, the strange quark was held fixed near its physical value while the degenerate light quarks were varied over a range of masses. (These are the same resources that were used to calculate the $\pi^{+} \pi^{+}$ scattering length described above.)

The scattering lengths of the five meson-baryon processes without annihilation have been calculated to $\mathscr{O}\left(m_{\pi, K}^{3}\right)$ in $S U(3) \mathrm{HB} \chi$-PT $[21,22]$, and are given by

$$
a_{\pi^{+} \Sigma^{+}}=\frac{1}{4 \pi} \frac{m_{\Sigma}}{m_{\pi}+m_{\Sigma}}\left[-\frac{2 m_{\pi}}{f_{\pi}^{2}}+\frac{2 m_{\pi}^{2}}{f_{\pi}^{2}} C_{1}+\mathscr{Y}_{\pi^{+} \Sigma^{+}}(\mu)+8 h_{123}(\mu) \frac{m_{\pi}^{3}}{f_{\pi}^{2}}\right]
$$




$$
\begin{gathered}
a_{\pi^{+} \Xi^{0}}=\frac{1}{4 \pi} \frac{m_{\Xi}}{m_{\pi}+m_{\Xi}}\left[-\frac{m_{\pi}}{f_{\pi}^{2}}+\frac{m_{\pi}^{2}}{f_{\pi}^{2}} C_{01}+\mathscr{Y}_{\pi^{+} \Xi^{0}}(\mu)+8 h_{1}(\mu) \frac{m_{\pi}^{3}}{f_{\pi}^{2}}\right] ; \\
a_{K^{+} p}=\frac{1}{4 \pi} \frac{m_{N}}{m_{K}+m_{N}}\left[-\frac{2 m_{K}}{f_{K}^{2}}+\frac{2 m_{K}^{2}}{f_{K}^{2}} C_{1}+\mathscr{Y}_{K^{+} p}(\mu)+8 h_{123}(\mu) \frac{m_{K}^{3}}{f_{K}^{2}}\right] ; \\
a_{K^{+} n}=\frac{1}{4 \pi} \frac{m_{N}}{m_{K}+m_{N}}\left[-\frac{m_{K}}{f_{K}^{2}}+\frac{m_{K}^{2}}{f_{K}^{2}} C_{01}+\mathscr{Y}_{K^{+} n}(\mu)+8 h_{1}(\mu) \frac{m_{K}^{3}}{f_{K}^{2}}\right] ; \\
a_{\bar{K}^{0} \Xi^{0}}=\frac{1}{4 \pi} \frac{m_{\Xi}}{m_{K}+m_{\Xi}}\left[-\frac{2 m_{K}}{f_{K}^{2}}+\frac{2 m_{K}^{2}}{f_{K}^{2}} C_{1}+\mathscr{Y}_{K^{0} \Xi^{0}}(\mu)+8 h_{123}(\mu) \frac{m_{K}^{3}}{f_{K}^{2}}\right],
\end{gathered}
$$

where the $C$ 's and the $h$ 's are low-energy constants (LECs) and the $\mathscr{Y}$ 's are loop functions given in Ref. [8]. It is clear that this is an overconstrained system. Many fitting strategies are possible, as discussed in Ref. [8]. We found it convenient to rewrite the chiral perturbation theory formulas as polynomial expansions. For instance, in the case of $\pi^{+} \Xi^{0}$ and $K^{+} n$, we formed the objects:

$$
\begin{aligned}
\Gamma_{N L O} & \equiv-\frac{4 \pi a f_{\phi}^{2}}{m_{\phi}}\left(1+\frac{m_{\phi}}{m_{B}}\right)=1-C_{01} m_{\phi} \\
\Gamma_{N N L O} & \equiv-\frac{4 \pi a f_{\phi}^{2}}{m_{\phi}}\left(1+\frac{m_{\phi}}{m_{B}}\right)+\frac{f_{\phi}^{2}}{m_{\phi}} \mathscr{Y}_{\phi B}\left(\Lambda_{\chi}\right)=1-C_{01} m_{\phi}-8 h_{1}\left(\Lambda_{\chi}\right) m_{\phi}^{2}
\end{aligned}
$$

for each of the processes. Notice that the left-hand sides of these equations are given entirely in terms of lattice-determined quantities, while the right-hand side provides a convenient polynomial fitting function. Figure 2 plots these functions for several of the processes. The shift of the value of $\Gamma$ from NLO to NNLO is dependent on the renormalization scale $\mu$, and therefore with the choice $\mu=\Lambda_{\chi}$ one would expect this shift to be perturbative if the expansion is converging. The large shifts in $\Gamma$ from NLO to NNLO are indicative of large loop corrections. The LECs fit to the lattice data are tabulated in Ref. [8]. While the NNLO LECs $h_{1}$ and $h_{123}$ appear to be of natural size, the NLO LECs $C_{0}$ and $C_{01}$ are unnaturally large. The extrapolated values of the five scattering lengths are given in Table 1 . While the $\pi^{+} \Sigma^{+}$and $\pi^{+} \Xi^{0}$ scattering lengths appear to be perturbative, the extrapolated kaon-baryon scattering lengths at NNLO deviate by at least $100 \%$ from the LO values. The seemingly inescapable conclusion is that the kaon-baryon scattering lengths are unstable against chiral corrections in the three-flavor chiral expansion, over the range of light-quark masses that we consider.

Given the poor convergence seen in the three-flavor chiral expansion due to the large loop corrections, it is natural to consider the two-flavor theory with the strange quark integrated out. In this way, $\pi \Sigma$ and $\pi \Xi$ may be analyzed in an expansion in $m_{\pi}$. To $\mathscr{O}\left(m_{\pi}^{3}\right)$ in the two-flavor chiral expansion, one has [23]

$$
\begin{array}{ll}
a_{\pi^{+} \Sigma^{+}}=\frac{1}{2 \pi} \frac{m_{\Sigma}}{m_{\pi}+m_{\Sigma}}\left[-\frac{m_{\pi}}{f^{2}}+\frac{m_{\pi}^{2}}{f^{2}} \mathrm{C}_{\pi^{+} \Sigma^{+}}+\frac{m_{\pi}^{3}}{f^{2}} h_{\pi^{+} \Sigma^{+}}^{\prime}\right], & h_{\pi^{+} \Sigma^{+}}^{\prime}=\frac{4}{f^{2}} \ell_{4}^{r}+h_{\pi^{+} \Sigma^{+}} ; \\
a_{\pi^{+} \Xi^{0}}=\frac{1}{4 \pi} \frac{m_{\Xi}}{m_{\pi}+m_{\Xi}}\left[-\frac{m_{\pi}}{f^{2}}+\frac{m_{\pi}^{2}}{f^{2}} \mathrm{C}_{\pi^{+} \Xi^{0}}+\frac{m_{\pi}^{3}}{f^{2}} h_{\pi^{+} \Xi^{0}}^{\prime}\right], & h_{\pi^{+} \Xi^{0}}^{\prime}=\frac{4}{f^{2}} \ell_{4}^{r}+h_{\pi^{+} \Xi^{0}},
\end{array}
$$




\begin{tabular}{ccccc} 
Quantity & LO (fm) & NLO fit (fm) & NLO (NNLO fit) (fm) & NNLO (fm) \\
\hline$a_{\pi \Sigma}$ & -0.2294 & $-0.208(01)(03)$ & $-0.117(06)(08)$ & $-0.197(06)(08)$ \\
$a_{\pi \Xi}$ & -0.1158 & $-0.105(01)(04)$ & $0.004(05)(11)$ & $-0.096(05)(12)$ \\
$a_{K p}$ & -0.3971 & $-0.311(18)(44)$ & $0.292(35)(48)$ & $-0.154(51)(63)$ \\
$a_{K n}$ & -0.1986 & $-0.143(10)(27)$ & $0.531(28)(68)$ & $0.128(42)(87)$ \\
$a_{K \Xi}$ & -0.4406 & $-0.331(12)(31)$ & $0.324(39)(54)$ & $-0.127(57)(70)$
\end{tabular}

Table 1: $S U(3)$ extrapolated scattering lengths. The first uncertainty in parentheses is statistical, and the second is the statistical and systematic uncertainty added in quadrature.

where $\ell_{4}^{r}$ is the LEC which governs the pion mass dependence of $f_{\pi}$ [13]. Note that the chiral logs have canceled, and in this form, valid to order $m_{\pi}^{3}$ in the chiral expansion, the scattering lengths have a simple polynomial dependence on $m_{\pi}$ [23]. Figure 3 shows the $68 \%$ and $95 \%$ confidence interval error ellipses in the $h$-C plane for both $\pi^{+} \Sigma^{+}$and $\pi^{+} \Xi^{0}$. Exploring the full $95 \%$ confidence interval error ellipse in the $h$-C plane yields

$$
\begin{aligned}
& a_{\pi^{+} \Sigma^{+}}=-0.197 \pm 0.017 \mathrm{fm} ; \\
& a_{\pi^{+} \Xi^{0}}=-0.098 \pm 0.017 \mathrm{fm} .
\end{aligned}
$$

These are the numbers that we quote as our best determinations of the pion-hyperon scattering
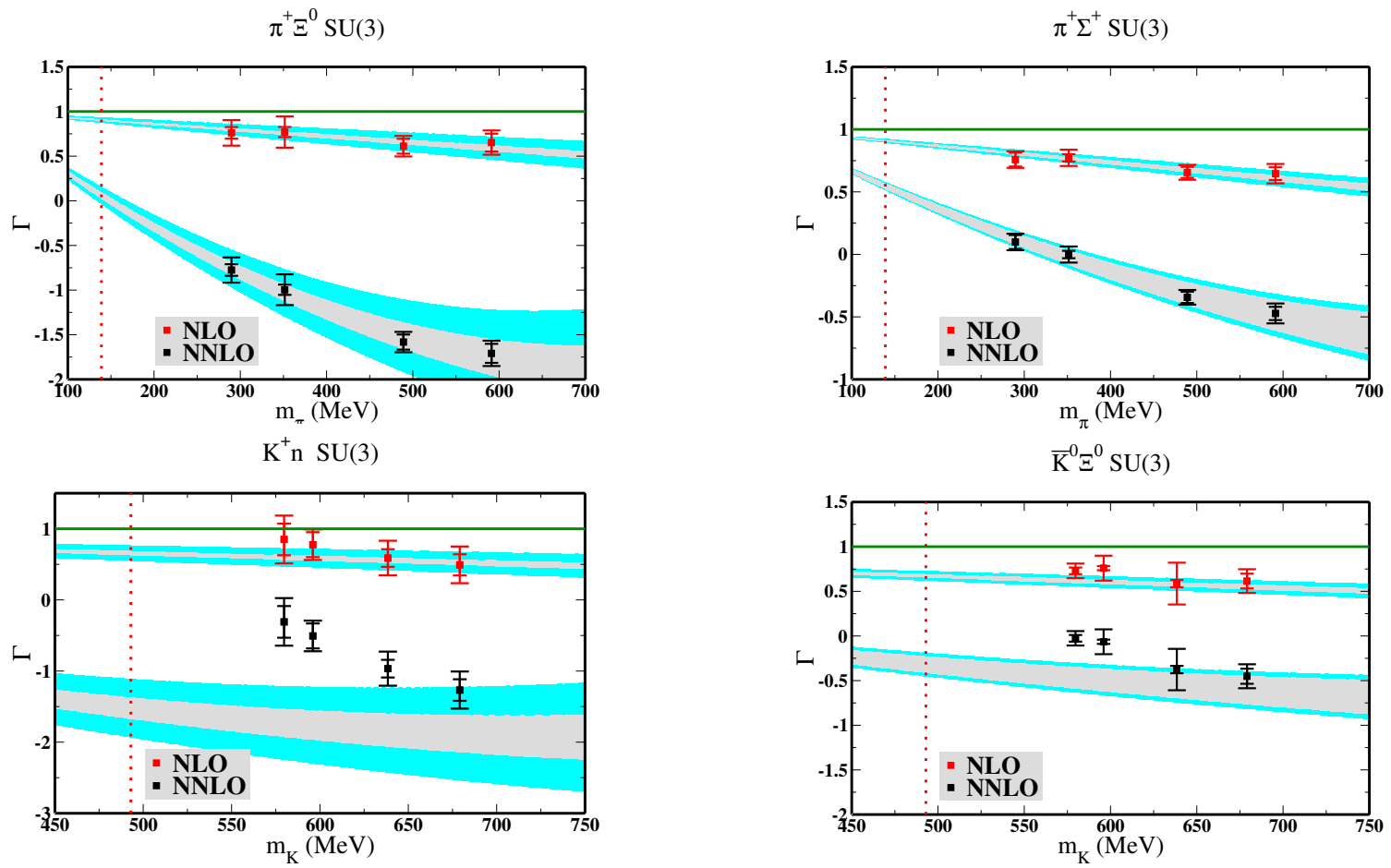

Figure 2: Plots of $\Gamma_{N L O}$ and $\Gamma_{N N L O}$ versus the Goldstone masses for four meson-baryon processes in the $\mathrm{SU}(3)$ case. The line at $\Gamma=1$ is the leading order curve, and dotted line is the physical meson mass. 
lengths. In Figure 4 we plot the scattering length versus the pion mass. (Note that the bar denotes
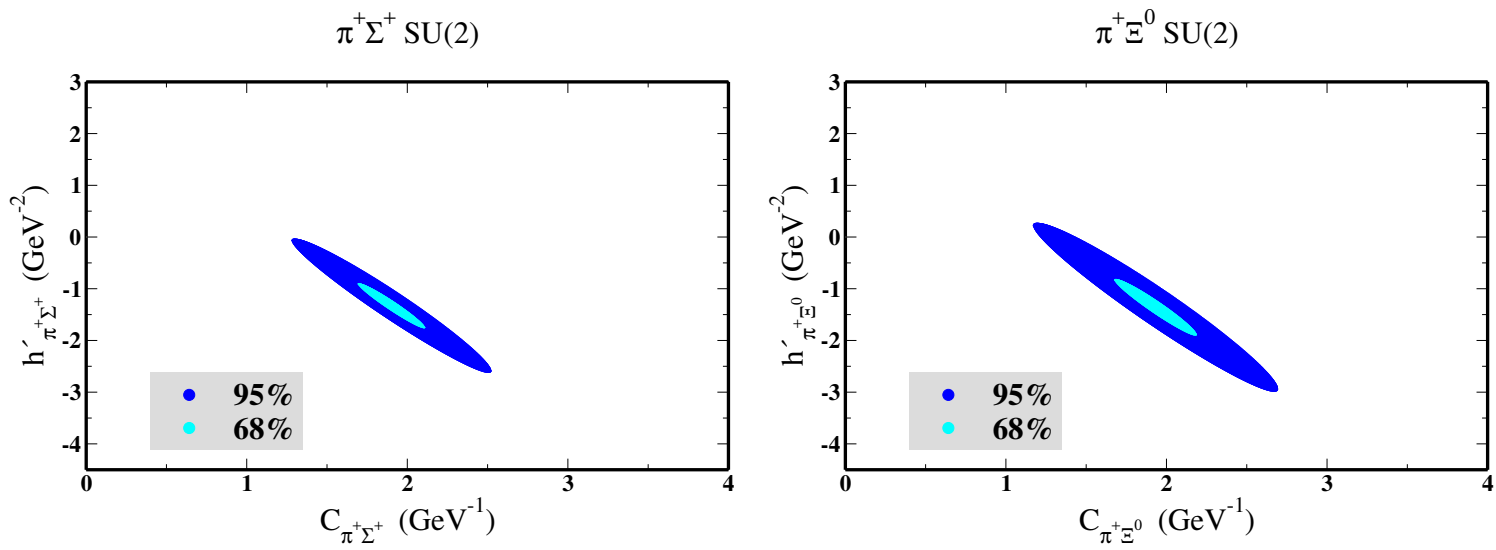

Figure 3: The 68\% (light) and 95\% (dark) confidence interval error ellipses for fits for the $\pi^{+} \Sigma^{+}$(left), and $\pi^{+} \Xi^{0}$ (right) processes.

the scattering length rescaled by a kinematical factor [8].)

$\bar{a}_{\pi^{+} \Sigma^{+}} \mathrm{SU}(2)$

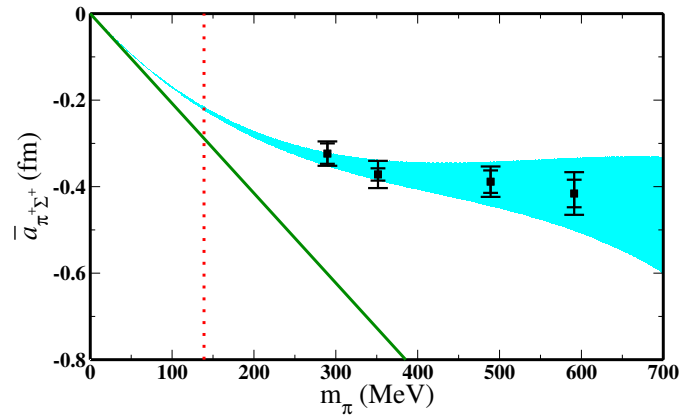

$\bar{a}_{\pi^{+} \Xi^{0}} \mathrm{SU}(2)$

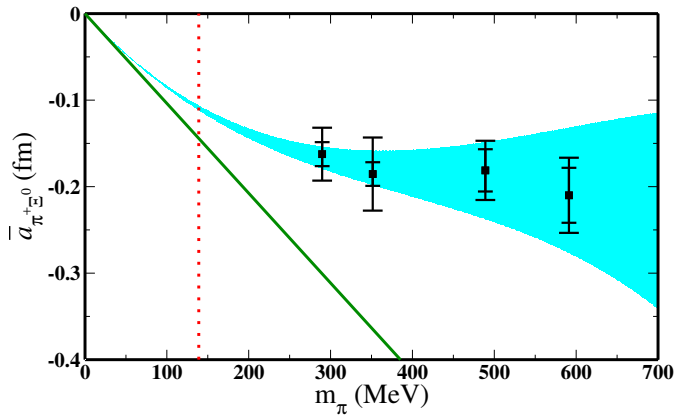

Figure 4: $\bar{a}$ plots for the $\pi^{+} \Sigma^{+}$, and $\pi^{+} \Xi^{0}$ processes versus the pion mass. The diagonal line is the leading order curve, and the dotted line is the physical pion mass. The innermost error bar is the statistical uncertainty and the outermost error bar is the statistical and systematic uncertainty added in quadrature. The filled bands are the fits to the LECs in the SU(2) case at NNLO.

\section{Baryon-Baryon Interactions}

The NPLQCD collaboration has performed the first full QCD calculations of nucleon-nucleon interactions [4] and hyperon-nucleon [5] interactions at low-energies with large pion masses. The nucleon-nucleon scattering lengths were found to be of natural size for the unphysically large values of the quark masses at which these calculations were performed. In contrast, the physical values of the scattering lengths are unnaturally large compared to the range of the nucleon-nucleon interaction. This suggests a fine-tuning of the underlying parameters of QCD (the quark masses and QCD scale) and lattice calculations with quark masses much closer to the physical values are needed to reproduce the experimental values. By contrast, very little is known about the interactions between nucleons and hyperons from experiment, and lattice QCD calculations can provide 
the best determinations of the corresponding scattering parameters and hence determine the role of hyperons in neutron stars.

The NPLQCD collaboration has undertaken an extensive exploration of the impact of highstatistics on one-, two- and three-baryon correlation functions on one ensemble of anisotropic clover gauge configurations that are presently being generated by the Hadron Spectrum Collaboration $[9,10]$. A total of $\sim 300,000$ sets of measurements were made using 1200 gauge configurations of size $20^{3} \times 128$ with an anisotropy parameter $\xi=b_{s} / b_{t}=3.5$, a spatial lattice spacing of $b_{s}=0.1227 \pm 0.0008 \mathrm{fm}$, and pion mass of $M_{\pi} \sim 390 \mathrm{MeV}$. The ground state baryon masses (in lattice units) were extracted with uncertainties that are at or below the $\sim 0.2 \%$-level. An example of the precision that has been obtained for single baryon masses with this calculation is shown in Figure 5, where the generalized effective mass plot of the $\Xi$ baryon is shown.

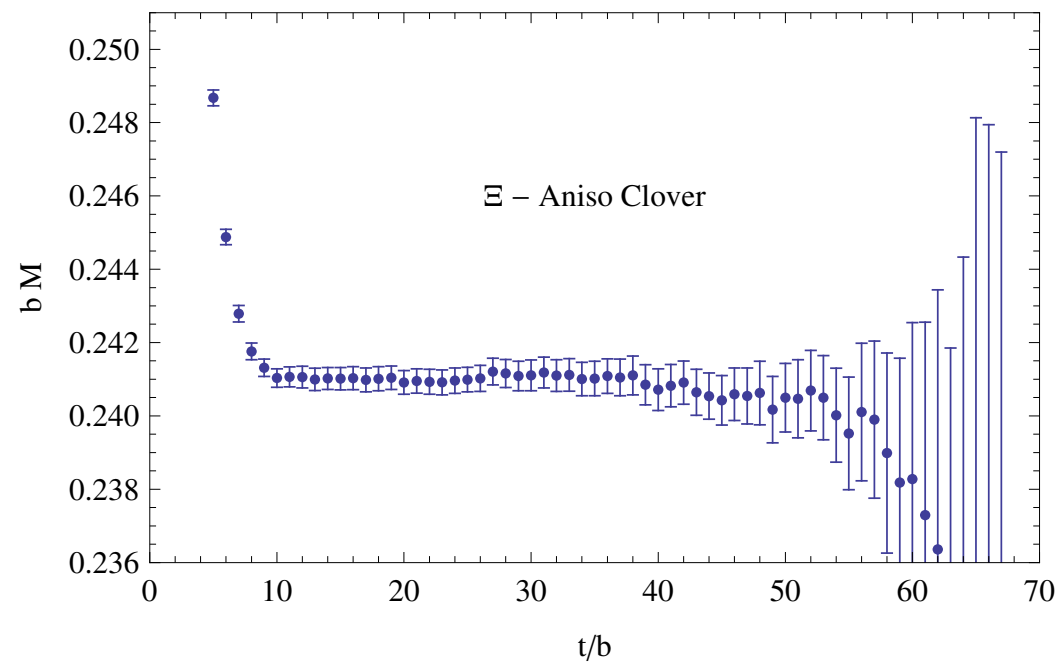

Figure 5: Effective mass plot for the $\Xi$ baryon from a high-statistics calculation using anisotropic clover gauge configurations.

With such statistics we were able to systematically explore the signal-to-noise issue in baryon systems, and found that, while signal/noise does degrades exponentially at large times, there is an intermediate time-interval for which it is time-independent, producing a noise-to-signal ratio per baryon that is essentially independent of baryon number. This is seen clearly in Figure 6. This feature greatly increases the number of nucleons that can be explored with lattice QCD for fixed computational resources. This was a significant finding and opens up the possibility of calculations with four and more baryons. In work that is currently in progress, we have obtained a number of phase-shifts in the two-baryon sector with $>5$ sigma-level significance. Figure 7 gives the energy shifts of various processes with associated statistical and systematic errors added in quadrature.

\section{Conclusion}

Lattice QCD calculations of two- and three-body interactions of pions and kaons are now a precision science (for those channels that do not involve disconnected diagrams). I have reviewed the first fully-dynamical lattice QCD calculation of meson-baryon scattering. An analysis of the 
scattering lengths of these two-body systems using $\mathrm{HB} \chi \mathrm{PT}$ has led to the conclusion that the threeflavor chiral expansion does not converge over the range of light quark masses that are investigated. By contrast, the $\pi^{+} \Sigma^{+}$and $\pi^{+} \Xi^{0}$ scattering lengths appear to have a well-controlled chiral expan-

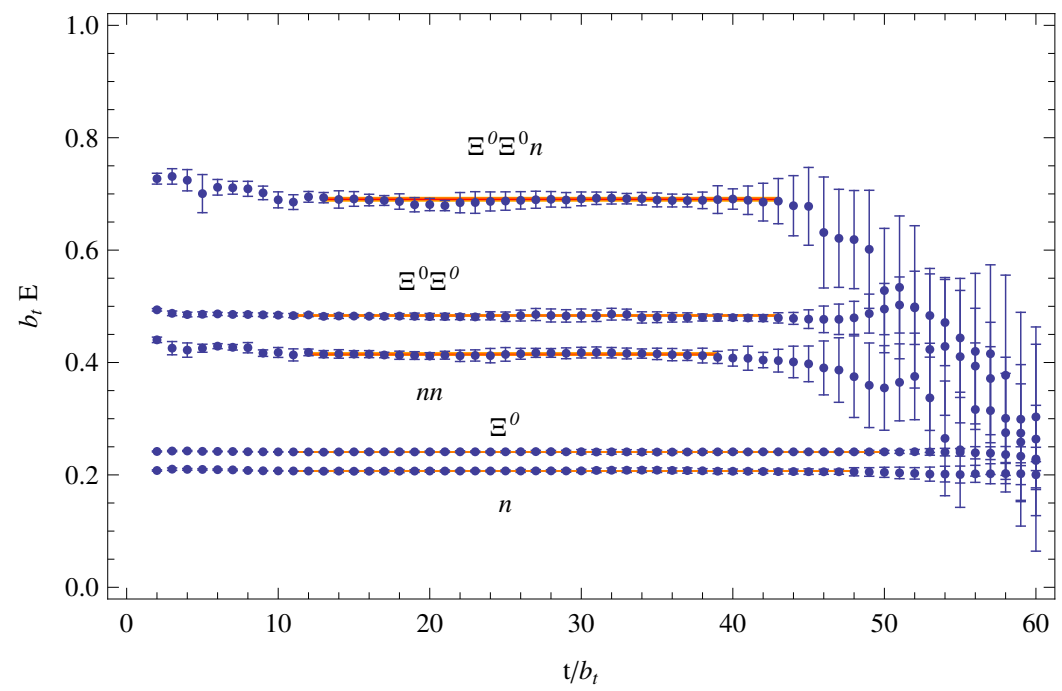

Figure 6: Effective mass plots for various one-, two- and three-body correlation functions calculated using anisotropic clover gauge configurations. There is an intermediate region of time slices for which signal/noise is time independent.

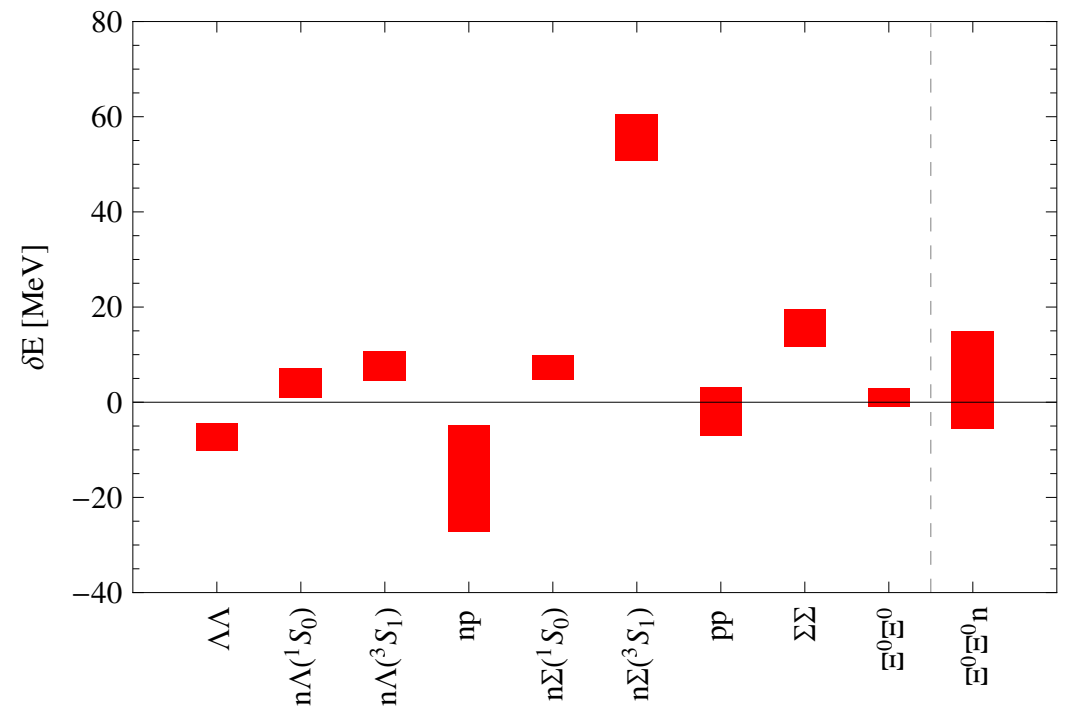

Figure 7: Energy splittings for various two- and three-body processes calculated using anisotropic clover gauge configurations. 
sion in two-flavor HB $\chi \mathrm{PT}$. Our results, $a_{\pi^{+} \Sigma^{+}}=-0.197 \pm 0.017 \mathrm{fm}$, and $a_{\pi^{+} \Xi^{0}}=-0.098 \pm 0.017$ $\mathrm{fm}$, deviate from the LO (current algebra) predictions at the one- and two-sigma level, respectively.

Clearly a milestone for this area of research would be to see a definitive signal for nuclear physics. In the case of the NN interaction, in addition to signal/noise issues, one must face a finetuned system that requires a non-perturbative effective field theory description. While this poses a tremendous challenge, this year has seen a great deal of progress in the NPLQCD collaboration's state goal to compute nuclear properties using lattice QCD. During the last year we performed a high-statistics calculation of one-, two- - and for the first time - three-baryon systems $[4,5]$, which represent a "jump" by an order of magnitude in the data volume produced in such calculations. The remarkable conclusion that the signal/noise problem is not as severe as previously thought promises to hasten a golden age of exploration for nuclear physics using lattice QCD.

\section{Acknowledgments}

I thank W. Detmold, T.C. Luu, K. Orginos, A. Parreño, M.J. Savage, A. Torok and A. Walker-Loud for fruitful collaboration. This work was supported by NSF CAREER Grant No. PHY-0645570.

\section{References}

[1] S. R. Beane, K. Orginos and M. J. Savage, Int. J. Mod. Phys. E 17, 1157 (2008) [arXiv:0805.4629 [hep-lat]].

[2] S. R. Beane, T. C. Luu, K. Orginos, A. Parreno, M. J. Savage, A. Torok and A. Walker-Loud, Phys. Rev. D 77, 014505 (2008) [arXiv:0706.3026 [hep-lat]].

[3] For a recent review, see V. Bernard, Prog. Part. Nucl. Phys. 60, 82 (2008) [arXiv:0706.0312 [hep-ph]].

[4] S. R. Beane, P. F. Bedaque, K. Orginos and M. J. Savage, Phys. Rev. Lett. 97, 012001 (2006) [arXiv:hep-lat/0602010].

[5] S. R. Beane, P. F. Bedaque, T. C. Luu, K. Orginos, E. Pallante, A. Parreno and M. J. Savage [NPLQCD Collaboration], Nucl. Phys. A 794, 62 (2007) [arXiv:hep-lat/0612026].

[6] G. P. Lepage, 'The Analysis Of Algorithms For Lattice Field Theory,' Invited lectures given at TASI' 89 Summer School, Boulder, CO, Jun 4-30, 1989. Published in Boulder ASI 1989:97-120 (QCD161:T45:1989).

[7] P. F. Bedaque and U. van Kolck, Ann. Rev. Nucl. Part. Sci. 52, 339 (2002) [arXiv:nucl-th/0203055].

[8] A. Torok et al., arXiv:0907.1913 [hep-lat].

[9] S. R. Beane et al., Phys. Rev. D 79, 114502 (2009) [arXiv:0903.2990 [hep-lat]].

[10] S. R. Beane et al., Phys. Rev. D 80, 074501 (2009) [arXiv:0905.0466 [hep-lat]].

[11] P. F. Bedaque and A. Walker-Loud, Phys. Lett. B 660, 369 (2008) [arXiv:0708.0207 [hep-lat]].

[12] S. Weinberg, Phys. Rev. Lett. 17, 616 (1966).

[13] G. Colangelo, J. Gasser and H. Leutwyler, Nucl. Phys. B 603, 125 (2001) [arXiv:hep-ph/0103088].

[14] H. Leutwyler, PoS C ONFINEMENT8, 068 (2008) [arXiv:0812.4165 [hep-ph]].

[15] S. M. Roy, Phys. Lett. B 36, 353 (1971). 
[16] J. W. Chen, D. O’Connell and A. Walker-Loud, Phys. Rev. D 75, 054501 (2007) [arXiv:hep-lat/0611003].

[17] D. B. Kaplan and A. E. Nelson, preprint HUTP-86/A023; Phys. Lett. B 175 (1986) 57; Phys. Lett. B 192, 193 (1987); Nucl. Phys. A 479, 273 (1988); Nucl. Phys. A 479, 285 (1988);

[18] M. Lu, M. B. Wise and M. J. Savage, Phys. Lett. B 337, 133 (1994) [arXiv:hep-ph/9407260].

[19] See, for instance, R. Babich, R. Brower, M. Clark, G. Fleming, J. Osborn and C. Rebbi, PoS LATTICE2008, 160 (2008) [arXiv:0901.4569 [hep-lat]].

[20] E. E. Jenkins and A. V. Manohar, "Baryon chiral perturbation theory using a heavy fermion Lagrangian," Phys. Lett. B 255, 558 (1991).

[21] Y. R. Liu and S. L. Zhu, Phys. Rev. D 75, 034003 (2007) [arXiv:hep-ph/0607100].

[22] Y. R. Liu and S. L. Zhu, Eur. Phys. J. C 52, 177 (2007) [arXiv:hep-ph/0702246].

[23] M. Mai, P. C. Bruns, B. Kubis and U. G. Meißner, arXiv:0905.2810 [hep-ph]. 